\title{
réflexion sur le béton compacté au rouleau
}

\author{
reflection on roller compacted contrete
}

\author{
P. LONDE* \\ Président honoraire de la Commission internationale des grands barrages \\ Ingénieur Conseil - Pierre Londe et Associés *
}

\section{Résumé}

Le B.C.R. est un nouveau béton faisant appel aux techniques de remblai pour sa mise en place.

Les utilisations faites à ce jour par les Américains et surtout les Japonais exploitent son caractère de béton et construisent avec le B.C.R. d'authentiques barrages-poids.

Dans d'autres utilisations, on se rapproche des remblais pour mettre en place des massifs ayant les propriétés d'un massif rocheux de qualité suffisante pour servir de fondation ou de parties d'ouvrage, comme à Tarbela, à Mosul ou à Kalabagh.

II semble que l'avenir du B.C.R. soit autre. II faut centrer la réflexion sur les formes nouvelles qu'il permettrait de réaliser et sur les possibilités offertes par l'association du B.C.R. avec des remblais ordinaires.

\section{Abstract}

The R.C.C. is a new material with the properties of concrete, but using the embankment techniques for placement.

The American and particularly the Japanese engineers use R.C.C. taking advantage of its "concrete" characteristics for construction of conventional gravity dams.

In other cases, the "embankment" aspects are emphazised for building man-made foundations or structures with the properties of a rock mass, such as at Tarbela, Mosul and Kalabagh.

It seems however that the future of R.C.C. could be contemplated in other directions. Consideration and thought should be given to the new dam design concepts that R.C.C. could make possible and to the feasibility of combining it with conventional earth and rock fills.

- Contribution à la journée d'étude E.D.F., Marseille, 26 novembre 1986.

- Pierre Londe et Associés, Tour Horizon, 52, quai de Dion-Bouton, 92806 Puteaux Cedex. 
1. Les deux techniques actuelles Roller Compacted Concrete (États-Unis) et Rolled Concrete for Dams (Japon) sont toutes deux appliquées au barrage-poids classique.

André COYNE disait, dès 1950 , que le problème majeur du béton était sa bonne vibration, une grande partie du ciment pouvant être économisée si l'on vibrait bien (problèmes de densité, ségrégation, ressuage...).

L'idée d'utiliser des rouleaux vibrants lourds, avec une énergie beaucoup plus élevée que celle des aiguilles traditionnelles, est à la base du Béton Compacté au Rouleau (B.C.R.).

A partir du moment où l'on emploie les rouleaux lourds, on est tenté d'introduire toute la technologie des remblais,

D'ailleurs, les premiers usages, par John LOWE III, étaient d'abord à Shimen (Taiwan), vers 1960, pour le noyau d'un batardeau en terre (manque d'argile, ajout de ciment dans du gravier sableux), puis à Tarbela (Pakistan), en 1975, pour des remplissages de fondation ou des remplacements de massifs rocheux défectueux. C'est encore pour constituer des fondations d'ouvrages annexes (prise d'eau, conduites forcées, évacuateur de crues, déversoir fusible) qu'on a eu recours, en 1983, à près d'un million de mètres cubes de B.C.R. au barrage de Mosul (Irak). Au barrage de Kalabagh (Pakistan), le projet actuel prévoit la mise en place de 1,5 million de metres cubes de B.C.R. pour fonder les 12 conduites forcées de $11 \mathrm{~m}$ de diamètre.

C'est au Japon qu'a été fait, en 1980, le premier barrage-poids en B.C.R. (appelé là-bas R.C.D.) : le barrage de Shimajigawa $\left(1655000 \mathrm{~m}^{3}\right)$. Tous les critères classiques du barrage-poids étaient respectés. Une économie de cout d'environ $10 \%$ a pu être dégagée.

A peu près simultanément, les Américains achevaient en 1983 le barrage-poids de Willow Creek en B.C.R. (appelé là-bas R.C.C.), mais avec un très faible dosage en ciment $\left(50 \mathrm{~kg} / \mathrm{m}^{3}\right)$ et la suppression des joints de contraction. Le profil du barrage restait celui du barrage-poids mais la mise en place du béton était très proche des techniques utilisées pour les barrages en terre. L'économie du coût était spectaculaire (environ $60 \%$ ) et la rapidité d'exécution sans précédent ( $315000 \mathrm{~m}^{3}$ en 4 mois). Cette belle première mondiale a toutefois souffert, pour que le mouvement soit largement suivi, du défaut d'étanchéité du barrage.

2. Depuis, on a beaucoup parlé de ces réalisations hardies, à la fois critiquées et enviées. Aujourd'hui, près d'une trentaine de barrages en B.C.R. sont en construction ou en projet dans le monde.

Le modèle japonais (R.C.D.) ne paraît pas faire autant école que le modèle américain (R.C.C.), si ce n'est qu'il rallie volontiers les timides, disons les prudents pour ne fâcher personne. En effet, il permet de ne pas se départir des critères caractérisant le barrage-poids. Mais il paraît évident que l'avenir est du côté du R.C.C., c'est-à-dire un nouveau matériau de remblai, nouveau en ce qu'il a, grâce au ciment, une cohésion inconnue jusqu'à maintenant dans les matériaux de remblai appelant un nouveau type de barrage.

Ce type de barrage pourrait être déduit de l'étude faite, il y a plus de 15 ans, par Jerry RAPHAËL et présentée par lui à la conférence d'Asilomar, 1970 (U.S.A.). Considérant le sol-ciment et son prix de l'époque, il a montré que le coût minimal était obtenu pour des fruits. sensiblement plus plats que ceux du barrage-poids. Il serait très intéressant de reprendre cette étude avec les prix actuels du B.C.R., en introduisant de très faibles. dosages en ciment. La hauteur du barrage (ou la valeur des contraintes) est un des paramètres de l'étude.

3. Un problème majeur se pose alors. Si l'on veut tirer le meilleur parti du faible coût du massif en B.C.R., en optimisant la section à un profil intermédiaire entre celui du barrage-poids et celui d'un remblai, on est nécessairement conduit à traiter l'étanchéité de l'ouvrage d'une manière séparée. En effet, une part importante du prix du B.C.R. actuel tient au soin couteux avec lequel on traite les joints entre reprises pour les rendre aussi peu perméables que possible. En outre le parement amont vertical du barrage-poids impose des coffrages et on en vient tout naturellement à placer du béton vibré classique contre ces coffrages.

L'adoption d'un fruit amont au moins égal à 0,75 (H)/1 (V) dispense des coffrages et du béton associé. On en arrive tout naturellement à construire un barrage en B.C.R. sans coffrages et sans joints, protégé à l'amont par un masque en béton armé, identique à celui qu'on pose sur la face amont des barrages en enrochement. Cette solution libère complètement de la recherche d'un B C.R. étanche, exactement comme pour les massifs en enrochement qu'on veut aussi perméables que possible. La figure 1 montre ce que pourrait être un tel barrage, avec déversement sur le parement aval. La partie déversante cependant peut être inspirée d'une solution souvent adoptée pour les barrages voutes, en laissant l'eau tomber en chute libre. La figure 2 schématise les dispositions, cette fois avec un coffrage aval, mais qui peut être sommaire et qui ne doit pas être étanche. Le fruit amont doit alors être à $1 / 1$ ou légèrement plus plat.

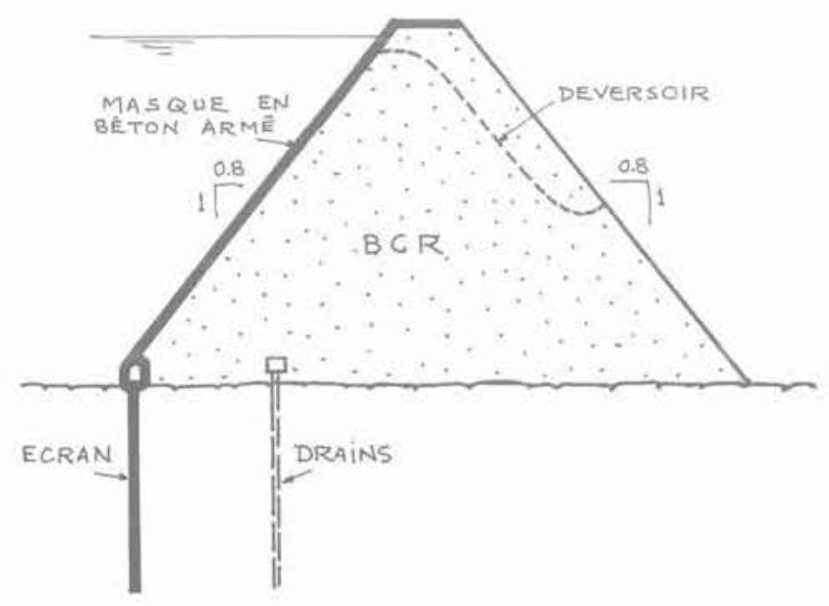

Fig. 1. - Barrage en BCR à profil symétrique. Fig. 1. - RCC dam with symmetrical section. 


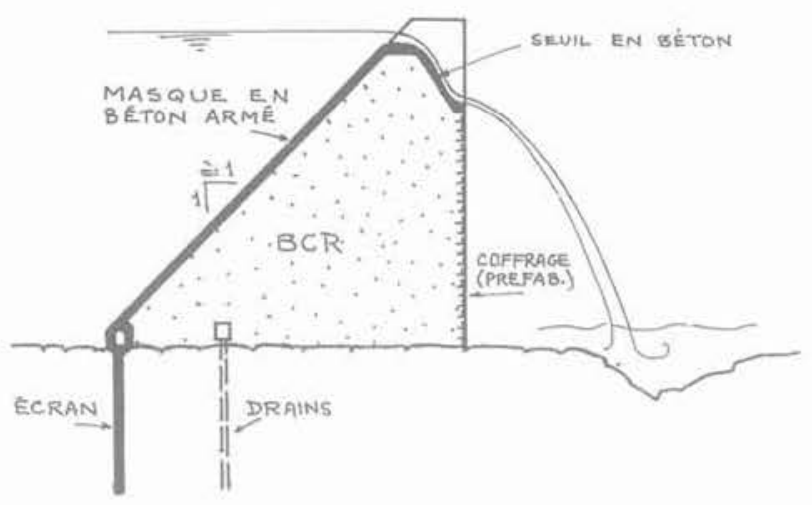

Fig. 2. - Barrage en BCR à déversoir en chute libre. Fig. 2. - RCC dam with free jump spillway.

4. Il est une autre voie de recherche. C'est celle des barrages composites dans lesquels on demande à une partie seulement, faite en B.C.R., de jouer son rôle de constituant à forte cohésion. L'adjonction de B.C.R. à un barrage en remblai classique permet de traiter élégamment les problèmes de protection pendant la construction et l'aménagement des déversoirs, car il résiste à l'érosion. L'adjonction de remblai classique à un barrage en B.C.R. résoud les problèmes de coffrage, ainsi que les sujétions d'accès. Elle permet aussi de traiter correctement l'étanchéité. Il semble que les projeteurs et les constructeurs n'aient pas encore compris tout ce que le B.C.R. permettra de faire, tant pour l'économie que pour la sécurité.

5. C'est dans cet esprit que travaille le Comité de la technologie des barrages de la C.I.G.B. (Commission internationale des grands barrages). Les solutions qui suivent sont prises parmi celles proposées par François Lempérière, membre du Comité. Elles méritent une réflexion approfondie.

\section{Solution I :}

Le B.C.R. est à l'aval, le remblai à l'amont.

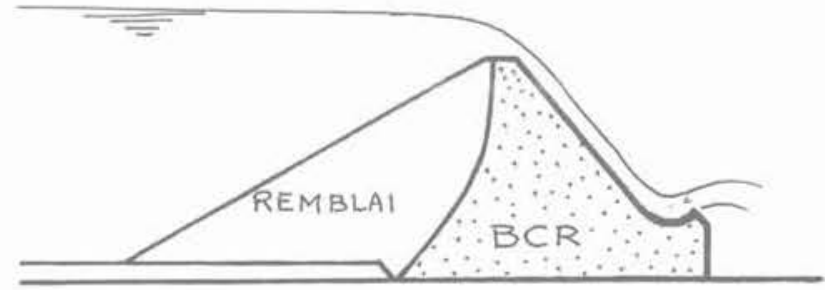

L'ensemble est déversant, il est construit sans coffrage. Le remblai stabilise l'ensemble, qui peut être construit sur une fondation à faible résistance au cisaillement. Le remblai donne un accès au B.C.R. à tous les niveaux. On peut disposer une couche étanche à l'interface des deux matériaux.

\section{Solution II :}

Variante de la précédente, pour les faibles hauteurs.

Le déversement est libre mais il faut coffrer la face aval verticale.

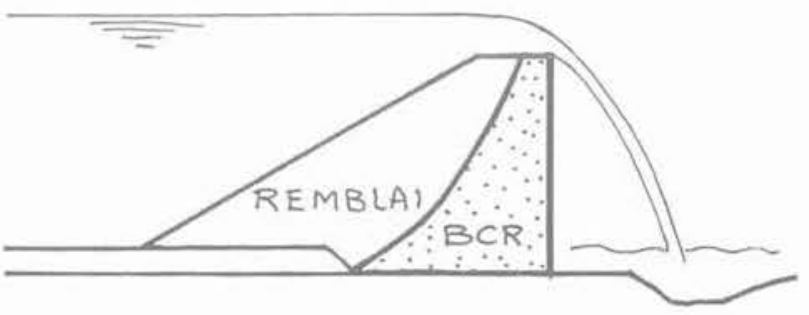

SOLUTION II

\section{Solution III :}

Inspirée du barrage de Zhaoshuge (Chine).

De nombreuses réalisations en Chine depuis 1958 : hauteur maximale de $32 \mathrm{~m}$, barrage courbe en plan, déversant sur un mur aval en maçonnerie, cœeur en enrochement, face amont en argile.

Ici on propose un mur aval en B.C.R. et un masque amont en béton armé (le problème du raccord entre masque et mur en B.C.R. est à étudier). Le cceur de l'ouvrage est un remblai en enrochement perméable. Cette solution est plus satisfaisante que les solutions I ou II.

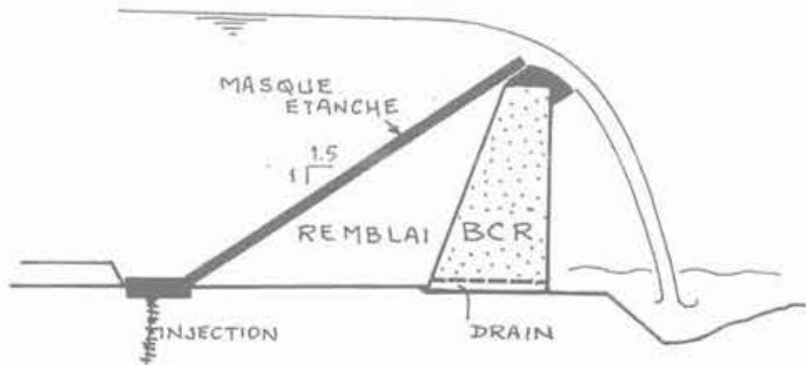

SOLUTION

111 


\section{Solution IV :}

C'est un barrage à deux zones (enrochement à l'aval, terre à l'amont) avec un déversoir en B.C.R., pour les faibles hauteurs.

Un rideau de palplanches peut être nécessaire, mais surtout pour la fondation dans le cas de terrain alluvial. Il faut autant de béton que dans une solution déversante classique, mais il est moins cher, sans coffrages, et l'accès est facile.
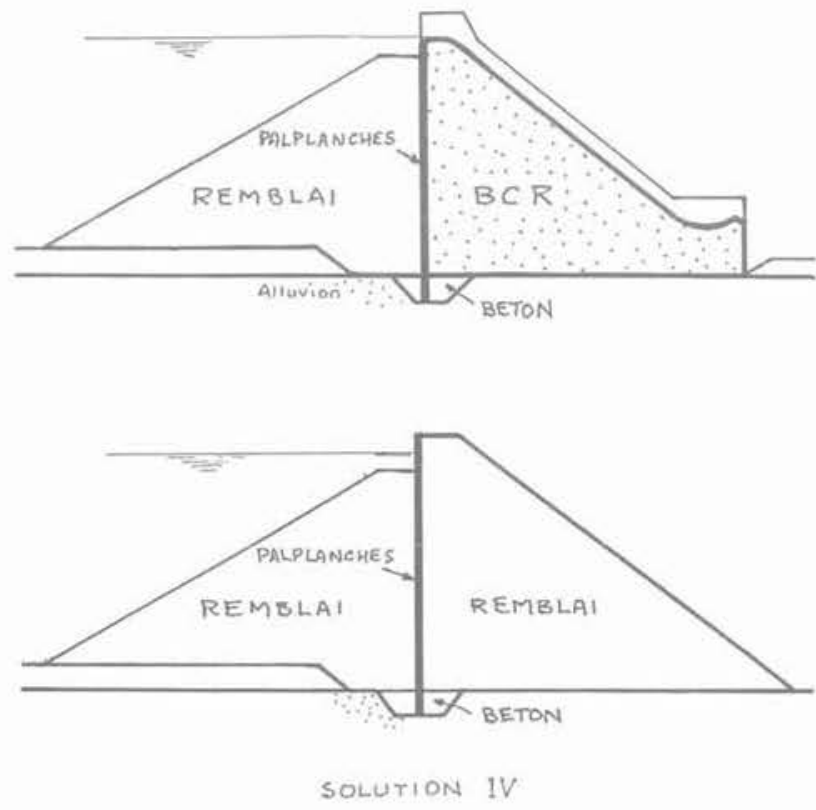

\section{Solution V:}

Cette solution est à rapprocher de la solution II mais avec des économies de ciment obtenues par la forme alvéolaire du B.C.R.

C'est un barrage à contreforts en B.C.R. dont les alvéoles sont remplis de remblai sans ciment. Il n'y a pas de coffrage, sauf au parement aval. Le volume de béton est moitié de celui du barrage-poids. Le déversement est facile et le passage de la crue de chantier est aisé. Cette variante n'est justifiée que pour les grandes hauteurs.

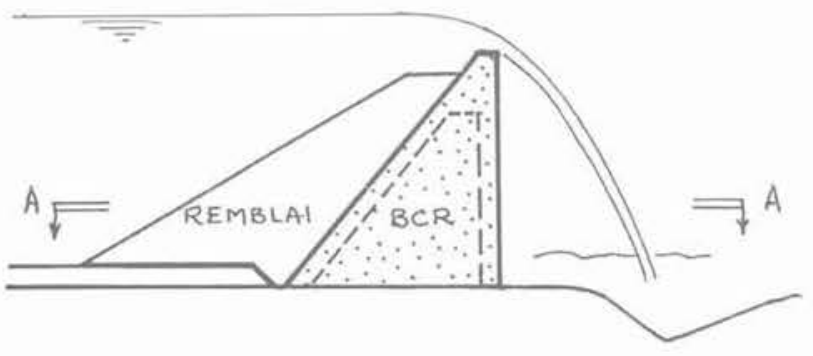

SECTION A-A

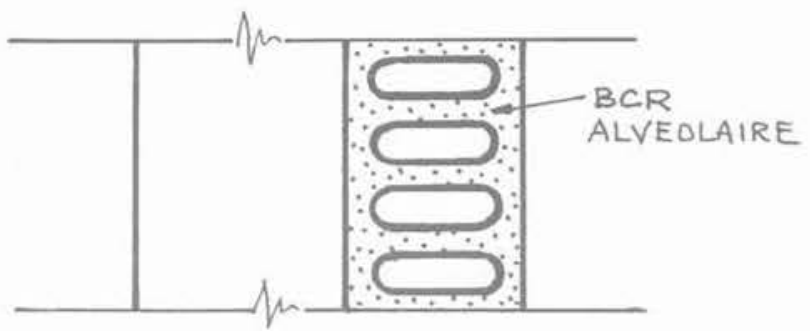

SOLUTION V

\section{Solution VI :}

A rapprocher du barrage à contreforts classique mais fait en B.C.R., sans coffrage, sauf à l'amont.

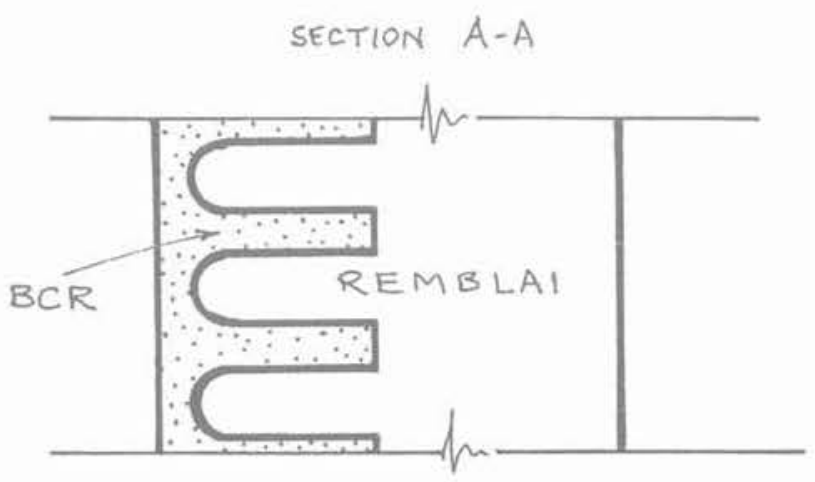

SOLUTION VI

\section{Solution VII :}

A rapprocher de la solution VI mais le coffrage amont est en voûtes minces armées. 
Le remblai développe sur les parois un effet de silo stabilisateur.

Cette énumération de barrages composites, tirant parti des qualités du B.C.R., ne se prétend pas exhaustive. Elle montre simplement que le mariage du B.C.R. et du remblai classique peut offrir des solutions très intéressantes et tout à fait dans la logique du B.C.R. qui se met en place comme un remblai.

SECTION B-B

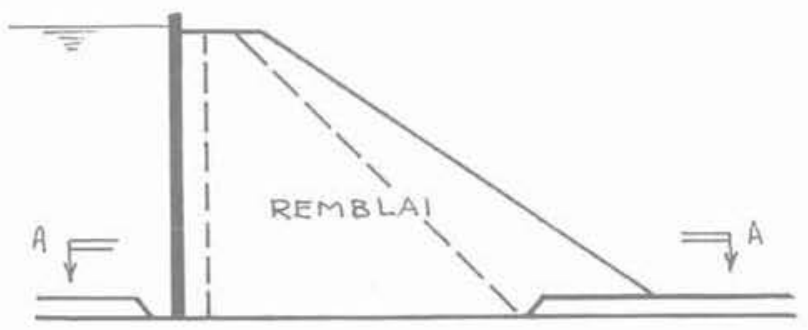

Il y a là matière à recherche, en associant la compétence des bureaux d'études et celle des entrepreneurs.

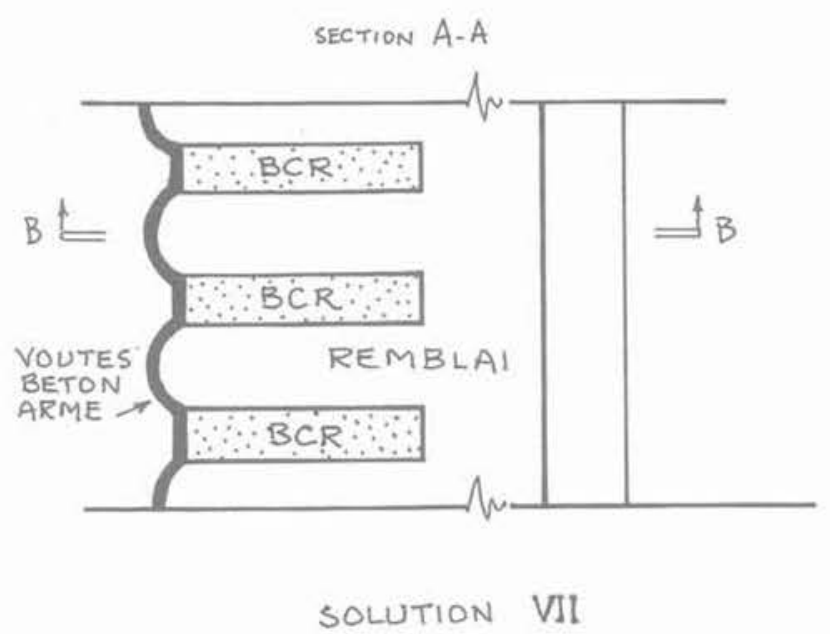

